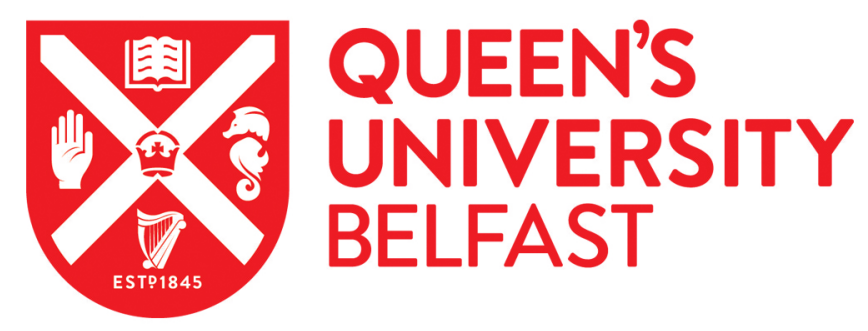

\title{
Inter-Technology Bridging Gateway: A Low Cost Legacy Adaptation Approach to Secure Industrial Systems
}

Khan, R., McLaughlin, K., Hastings, J., Laverty, D., \& Sezer, S. (2018). Inter-Technology Bridging Gateway: A Low Cost Legacy Adaptation Approach to Secure Industrial Systems. In 2018 Power and Energy Society General Meeting (PESGM) (IEEE Power \& Energy Society General Meeting). Institute of Electrical and Electronics Engineers Inc..

Published in:

2018 Power and Energy Society General Meeting (PESGM)

Document Version:

Peer reviewed version

Queen's University Belfast - Research Portal:

Link to publication record in Queen's University Belfast Research Portal

Publisher rights

() 2018 IEEE. This work is made available online in accordance with the publisher's policies. Please refer to any applicable terms of use of the publisher.

\section{General rights}

Copyright for the publications made accessible via the Queen's University Belfast Research Portal is retained by the author(s) and / or other copyright owners and it is a condition of accessing these publications that users recognise and abide by the legal requirements associated with these rights.

Take down policy

The Research Portal is Queen's institutional repository that provides access to Queen's research output. Every effort has been made to ensure that content in the Research Portal does not infringe any person's rights, or applicable UK laws. If you discover content in the Research Portal that you believe breaches copyright or violates any law, please contact openaccess@qub.ac.uk. 


\title{
Inter-Technology Bridging Gateway: A Low Cost Legacy Adaptation Approach to Secure Industrial Systems
}

\author{
Rafiullah Khan, Kieran McLaughlin, John Hastings, David Laverty and Sakir Sezer \\ Queen's University Belfast, Belfast, United Kingdom \\ Email: \{rafiullah.khan, kieran.mclaughlin, j.hastings, david.laverty, s.sezer\}@qub.ac.uk
}

\begin{abstract}
A major hindrance in industry modernization is the interoperability issues between existing legacy and new systems. Legacy systems normally have security vulnerabilities and are cost prohibitive to upgrade/replace. Thus, a feasible solution is needed to equip legacy systems with secure emerging technologies. This paper presents a low-cost ARM processor based Inter-Technology Bridging Gateway (ITBG). Its modular design makes it easily customizable for any industrial application. As a use case, this paper presents design, implementation, compliance testing and validation of ITBG for synchrophasor technology in smart grid. It bridges technological gap between legacy and new phasor devices by supporting two-way protocol translation between IEEE C37.118.2 and IEC 61850-90-5 standards. Further, it ensures system security by using IEC recommended GDOI security mechanism. Experiments in a laboratory based physical testbed successfully validated ITBG for different time-critical synchrophasor applications.
\end{abstract}

\section{INTRODUCTION}

Industry 4.0 promises increased productivity, high efficiency, quality and countless value creation opportunities. However, industry modernization is a challenging process as highlighted in Fig. 1. Unlike consumer IT devices, industrial devices are expensive with high deployment complexity, usually have no security patches/updates and build to last for several decades. Thus, existing legacy systems are expected to remain as part of the modernized industry. Their supported legacy protocols are plagued by cyber security and data privacy issues. Further, legacy systems face interoperability and integration issues with new systems.

Particularly, security and legacy adaptation challenges exist in smart grid domain. Power companies have deployed a large number of expensive phasor devices (i.e., Phasor Measurement Units (PMUs) and Phasor Data Concentrators (PDCs)) over last decade. They are used in synchrophasor technology for Wide-Area Monitoring, Protection And Control (WAMPAC). Typically, legacy devices are IEEE C37.118.2 compliant and lack security features. Recently, IEC 61850-90-5 emerged as new communication framework with security support [1]. However, legacy phasor devices are critical assets of power companies and difficult to upgrade due to cost and installation complexity. Further, integration of modern IEC 61850-90-5 compliant phasor devices in power grids raises interoperability issues with legacy IEEE C37.118 compliant devices and applications. Thus, a low cost solution is needed to enable legacy and new systems to interoperate securely.

\section{A. Related Work}

Several researchers have identified challenges in industry modernization with a prime focus on security and legacy adaptation [2]-[4]. Authors in [5] identified legacy systems as a root cause behind successful industrial cyber security incidents in past. Similarly, legacy devices in power grids also have security vulnerabilities as identified in [6], [7]. Thus, ensuring absolute security is a difficult job unless legacy systems are updated or replaced [8], [9].

Authors in [10], [11] addressed legacy adaptation by providing connectivity. Thus, legacy devices can be remotely accessed and controlled. However, presented works do not address security and interoperability between legacy and new devices using different standards. A similar case study on enabling connectivity to legacy devices is presented in [12].

To equip legacy devices with security features, authors in [13] proposed a security middleware. It runs on both, the legacy device and its control application. It provides mutual authentication and integrity/legitimacy checks on all data exchanges with industrial devices. However, the presented work focuses on security and does not address interoperability issues.

To address integration issues, manufacturers must strictly follow relevant standards [2]. Firouzi et al. [14] presented a gateway that translates packets from IEEE C37.118.2 standard into IEC 61850-90-5 standard. However, one-way translation enables IEEE C37.118.2 compliant devices to be used in IEC 61850-90-5 compliant system but not vice versa. To properly address interoperability, two-way translation is necessary. Further, their work lacks security features.

\section{B. Paper Contributions}

This paper presents a plug \& play Inter-Technology Bridging Gateway (ITBG) to address legacy adaptation and security issues in industry modernization. Unlike previous works [10], [11], the scope of the proposed ITBG is not to provide remote controllability to legacy devices. Instead, ITBG eliminates technological gap between legacy and new systems which were manufactured using different communication standards. The ITBG is a major step beyond the most relevant previous work [14] as it: (i) supports two-way protocols/standards translation feature, (ii) embeds security features for protection against cyber attacks, (iii) can work with multiple phasor 


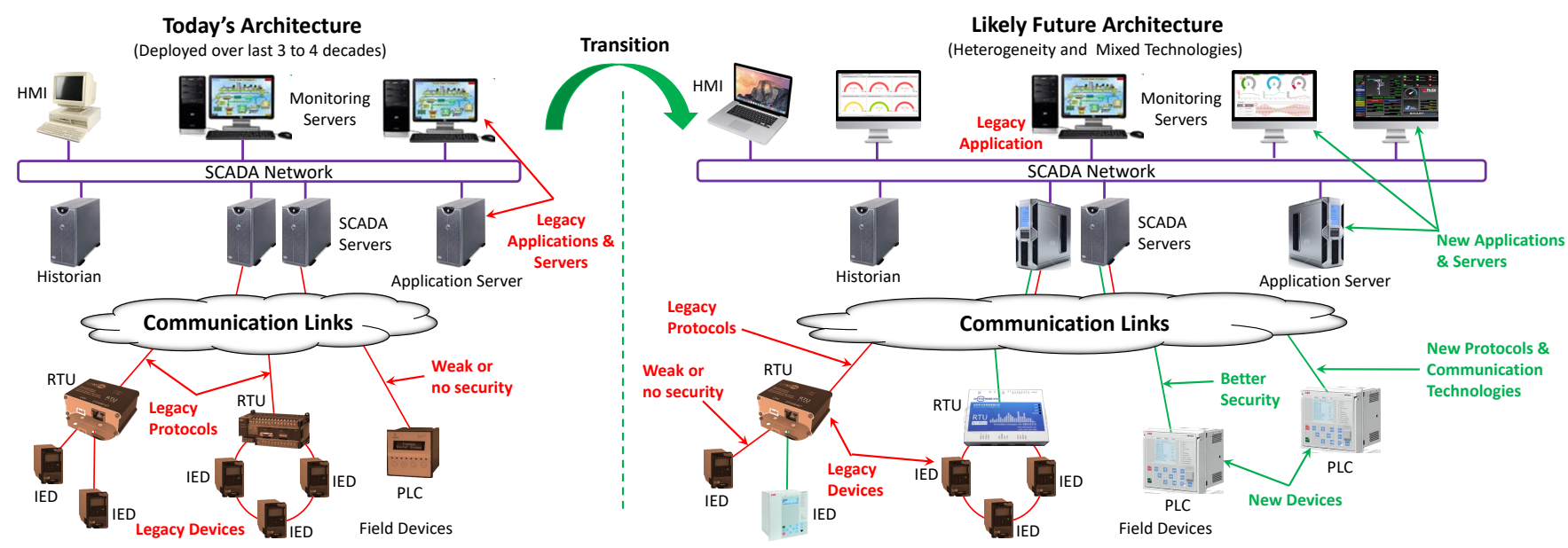

Figure 1. The modernization of an industry. New device and applications are expected to co-exist raising interoperability, integration and security challenges.

devices (PMUs/PDCs) and (iv) has modular architecture and easily customizable for any industrial application.

This paper presents the architectural design of an ITBG. As a use case, implementation details are provided for synchrophasor technology in smart grid. It provides two-way translation between IEEE C37.118.2 and IEC 61850-90-5 standards to enable interoperability and integration between legacy and new phasor devices/applications. Before incorporating in ITBG, both implemented IEEE C37.118.2 and IEC 6185090-5 libraries were compliance tested with PMU Connection Tester for functional validation. To protect synchrophasor communication from cyber attacks, the IEC recommended Group Domain Of Interpretation (GDOI) security mechanism is implemented which uses dynamic security credentials and policies. This paper also presents experimental evaluation of the ITBG in a physical laboratory based testbed and validates its suitability on a low-cost ARM processor for synchrophasor applications with strict data rate and latency requirements.

\section{Proposed Inter-Technology Bridging Gateway}

Legacy industrial devices use obsolete technologies/standards, lack security features and are cost prohibitive to replace. To avoid risk of breaking industrial control systems, devices once installed often have no software updates/patches. Further, they typically have several decades long life cycle. Therefore, industry modernization has to tackle with challenges emerging due to co-existence of legacy and new devices/applications.

\section{A. ITBG Concept}

The objective of the ITBG is to enable smooth industry modernization by addressing system scalability, interoperability and security challenges. ITBG is a small low-cost device that can be plugged to a legacy and/or new device/application. Its aim is to act as technology bridge between legacy and new devices/applications. Fig. 2 depicts the basic concept highlighting three scenarios: (i) It translates obsolete communication technology of legacy device into latest technology supported by the new application (i.e., ITBG-A), (ii) It translates latest communication technology of new device into obsolete technology supported by the legacy application (i.e., ITBG-C), and (iii) It secures communication between legacy device and legacy application by using latest secure technologies (i.e., ITBG-B and ITBG-C). Scenarios i \& ii need one ITBG device whereas scenario iii needs two ITBG devices. As shown in Fig. 2, the proposed ITBG solution does not require any replacement in existing industrial infrastructure. The only requirement is to attach an inexpensive plug \& play ITBG with two network interfaces so that all network traffic passes through it.

\section{B. Basic Functional Blocks}

Based on the highlighted capabilities in Fig.2, the ITBG needs two basic functional blocks:

1) Protocol Translator: ITBG needs the implementation of communication protocols used by legacy and new devices/applications. Based on the protocols, ITBG then needs to implement their inter-conversion features. Unlike IT networks, fewer protocols are used in industries. Certain protocols e.g., Modbus and DNP3 are used in several industries so they all can benefit from same ITBG implementation.

2) Communication Security: Most industrial protocols lack security features e.g., IEEE C37.118.2, Modbus, etc. However, certain protocols have security support e.g., IEC 61850-905. Thus, ITBG needs to implement security functionalities for non-secure protocols. It needs to be the part of industrial security system or use its own security mechanism in certain scenarios (e.g., ITBG-B and ITBG-C link security in Fig. 2). Not essential for legacy adaptation, it may also implement intrusion detection capabilities. Thus, ITBG can be part of very effective distributed Intrusion Detection System (IDS) with several benefits as highlighted in [15].

\section{Integration in Industrial Systems}

There are two integration/deployment possibilities for ITBG as highlighted in Fig.2: on-board ITBG and network ITBG. On-board ITBG is a software package executed on the industrial device or application PC. It does not require dedicated 


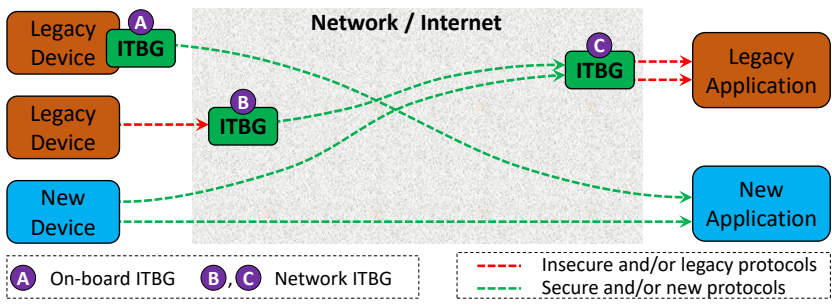

Figure 2. ITBG concept and deployment possibilities.

hardware but its service is limited to a single device/application. On-board ITBG may easily run on the same PC as the industrial application but could be challenging to execute on legacy devices. Whereas, network ITBG can simultaneously offer service to multiple devices and applications. It may or may not require a dedicated standalone hardware. E.g., ITBG software can be executed on existing network switches or gateway devices. In case of standalone network ITBG, a lowcost device may be used e.g., Raspberry Pi. Hybrid on-board and network ITBG solution may also be used by industries.

\section{USE CASE: SyNChrophasor TEChNOLOGY IN SMART GRID}

This paper focuses on synchrophasor technology as the use case implementation of ITBG. Synchrophasor technology was first introduced in 1980s and initial phasor devices became available in late 1990s. Power companies have already deployed large number of expensive legacy phasor devices (PMUs, PDCs) which use obsolete insecure communication technologies. Over time, several WAMPAC applications have been proposed using synchrophasor technology. However, most real-time control applications are still in laboratory validation and lack of security is a key concern for their deployment in real grid. For reliable and trustworthy WAMPAC operations, replacing or equipping legacy phasor devices with latest secure technologies is of paramount significance. Thus, ITBG can play a vital role in the modernization of legacy synchrophasor-based systems.

\section{A. Communication Standards}

IEEE 1344 is the first communication standard developed for synchrophasors in 1995. It was replaced by IEEE C37.118 in 2005. After minor modifications, IEEE C37.118.2 emerged as the most recent version of IEEE standard in 2011. All these different IEEE standards lack security features and are vulnerable to cyber attacks [6]. To tackle with security issues and harmonize communication with IEC 61850 utility automation standard, IEC 61850-90-5 was published in 2012. IEC 61850-90-5 supports security features but its use in power systems is still very limited [1]. A main reason behind this is the interoperability and integration issues with the existing legacy phasor devices and applications.

\section{B. GDOI: Group-based Security Mechanism}

GDOI is published in RFC 6407 and is recommended security mechanism by IEC for synchrophasor-based systems. It is supported in IEC 61850-90-5 standard. Fig. 3 depicts GDOI

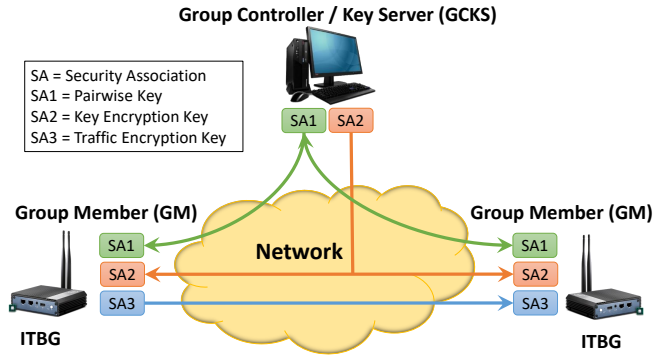

Figure 3. Functional overview of GDOI security mechanism used by ITBG.

functional overview. It consists of two types of devices: Group Controller/Key Server (GCKS) and Group Member (GM). GCKS manages security policies and credentials for a group of GMs and updates them periodically. GDOI consists of two phases: (i) authentication and (ii) security credentials update. Authentication phase is based on certificates or any public key cryptography technique using ISAKMP protocol. Security credentials update has two types of exchanges: GroupKeyPull and GroupKey-Push. GroupKey-Pull is used by a newly joined GM to acquire updated security credentials from GCKS. Whereas, GroupKey-Push is used by GCKS to periodically push security credential updates to all authorized GMs.

As depicted in Fig. 3, GDOI consists of three types of Security Associations (SA). Pairwise key (SA1) is specific to each GM and established during authentication phase. It protects GroupKey-Pull exchange during which a GM acquires Key Encryption Key (KEK) and Traffic Encryption Key (TEK) from GCKS. KEK SA is used to protect GroupKey-Push exchange. Whereas, TEK SA is used by GMs to protect communication among each other. Both, KEK and TEK have assigned validities and replaced periodically by GCKS. The authentication and freshness features of GDOI makes it a strong security mechanism against cryptanalysis attacks.

\section{ITBG for Synchrophasor-based Systems}

To address legacy adaptation, the ITBG needs to implement synchrophasor communication standards and their interconversion features. To ensure secure and trustworthy communication, the ITBG needs to implement GDOI client software and acts as a group member of GCKS (see Fig. 3). Fig. 4 depicts network ITBGs acting as secure proxy between legacy IEEE C37.118.2 compliant PMU and WAMPAC application. Each ITBG supports two-way translation features between relevant standards and embeds a GDOI client for security features. Based on the standard, ITBG exchanges certain number of packets with PMU to establish communication. Such semantics are explained in relevant communication standards and briefly described in [1].

Most commercially available phasor devices support either IEEE C37.118.2 or insecure IEC 61850-90-5 (without GDOI). Thus, ITBG should be able to work in four possible cases: (i) PMU/PDC is IEEE C37.118.2 compliant, (ii) WAMPAC application is IEEE C37.118.2 compliant, (iii) PMU/PDC is IEC 61850-90-5 compliant and (iv) WAMPAC application is IEC 61850-90-5 compliant. Further, a single ITBG needs to 


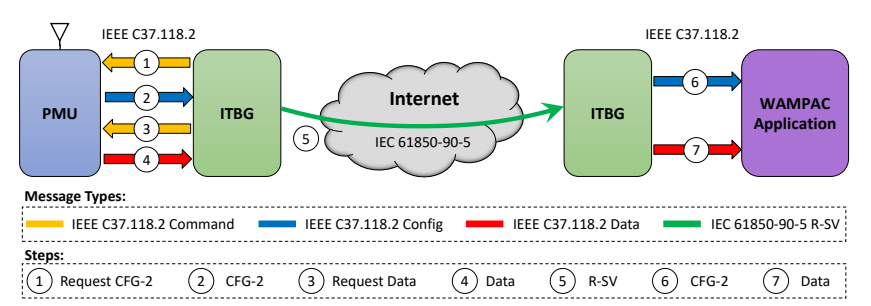

Figure 4. ITBGs enabling IEEE C37.118.2 compliant legacy PMU and WAMPAC application to securely communicate using IEC 61850-90-5.

be able to offer services simultaneously to multiple phasor devices (PMUs/PDCs).

\section{IMPLEMENTATIONS}

The ITBG software is implemented in Linux OS using Python programming language and PyCrypto libraries. The core of ITBG software consists of six modules as depicted in Fig. 5. Application Manager is responsible for managing and coordinating activities among different modules and to enable an operator to control/configure the software. Security Mechanism contains GDOI client that is used to authenticate with GCKS and acquire security policies and credentials. The authentication process in current implementation is based on Diffie Hellman public key cryptography technique using ISAKMP protocol. Encryption Algorithms module provides encryption/decryption methods for three algorithms: AES-256-CBC, AES-256-GCM and AES128-GCM. Signature Algorithms module provides signature calculation/verification methods for seven algorithms: AES-GMAC128, AES-GMAC64, HMAC-SHA512, HMACSHA384, HMAC-SHA256, HMAC-SHA256-128 and HMACSHA256-80. These algorithms are used by GDOI client as well as IEC 61850-90-5 communication framework. The choice of algorithm and security credentials is decided by GCKS and updated periodically. Protocol Translator contains complete implementation of IEEE C37.118.2 and IEC 61850-90-5 libraries in Python and provides their two-way inter-conversion features. Task Scheduler module is responsible to execute a task at its intended time instant, check validity of security credentials and perform other periodic events.

\section{TEST-BED AND EXPERIMENTAL EVALUATION}

The testbed for functional evaluation of ITBG for a synchrophasor-based system is depicted in Fig. 6. It consists of a commercial PMU from ABB, an OpenPMU [16], custom implemented emulated PMU and PDC (ePMU/ePDC) and a monitoring application. All except the commercial PMU support IEEE C37.118.2 as well as IEC 61850-90-5. All PMUs are configured to include three integer phasors in polar format, frequency and Rate-Of-Change-Of-Frequency (ROCOF) in integer format, two analog float values and one digital word. The implemented ITBG software is light-weight (20.53 MB) and executed on inexpensive ARM-based Raspberry Pi v2. Secure communication is only between ITBGs that passes through NRL CORE (emulated but similar to realistic network).

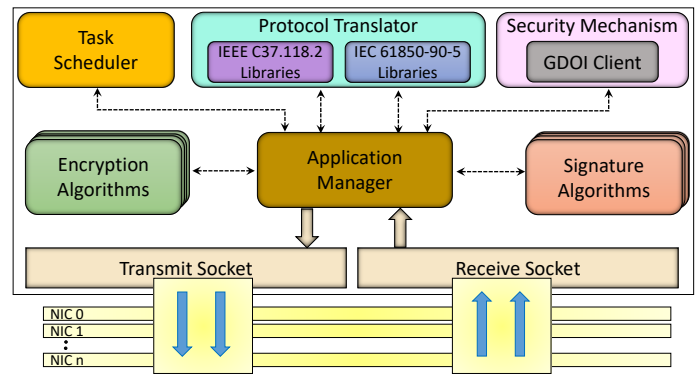

Figure 5. Basic modular architectural design of ITBG for synchrophasorbased system. It supports GDOI based security mechanism and two-way translation between IEEE C37.118.2 and IEC 61850-90-5 standards.

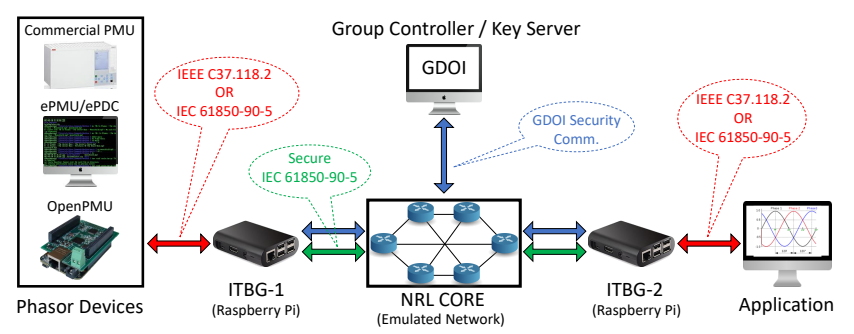

Figure 6. Experimental testbed for the validation of ITBG features.

The first step in evaluation is functional verification and compliance testing of the ITBG. To this aim, PMU Connection Tester tool was used. It successfully decoded and visualized the ITBG output indicating correctness of protocol translation features. GDOI security package, ePMU and ePDC were functionally verified using the Wireshark packet capturing tool. ITBG was able to successfully authenticate with GDOI GCKS and acquire security credentials. By analyzing network traffic in Wireshark, it was verified that security credentials change on periodic basis.

The next step is to measure performance critical factors (latencies, supported transmission rate and bandwidth requirement) which may impact real-time operations in synchrophasor network. The aim of such experiments is to investigate if a compact low-cost ARM-based Raspberry $\mathrm{Pi}$ is suitable to employ ITBG. Table I presents latencies (averaged over 100 trials) for ITBG communication with GCKS during different phases of GDOI. The latencies are quite small. Thus, GCKS can authenticate and perform GroupKey-Pull with up to 5 ITBGs per second. This is quite good number knowing that authentication and GroupKey-Pull are performed only once by each ITBG. GCKS is configured to push new security credentials 5 seconds before the expiry of old credentials,

Table I

PRocessing LATENCIES OF GDOI SECURITY PACKAGE.

\begin{tabular}{lcccc}
\hline \multirow{2}{*}{ GDOI Phase } & \multicolumn{4}{c}{ Processing Latencies (ms) } \\
\cline { 2 - 5 } & Min & Avg & Max & Std. Dev \\
\hline Authentication & 106.83 & 129.76 & 154.52 & 3.01 \\
GroupKey-Pull & 85.79 & 110.24 & 123.53 & 2.79 \\
GroupKey-Push & 16.14 & 21.96 & 24.78 & 1.31 \\
\hline
\end{tabular}

Values are averaged over 100 trials and include ITBG plus GCKS processing latencies. 
Table II

EXPERIMENTAL RESULTS OF ITBG FOR DIFFERENT TYPES OF SYNCHROPHASOR APPLICATIONS.

\begin{tabular}{|c|c|c|c|c|c|c|c|}
\hline \multirow{2}{*}{$\begin{array}{l}\text { Application } \\
\text { Criticality }\end{array}$} & \multirow{2}{*}{$\begin{array}{c}\text { Required } \\
\text { Rate }\end{array}$} & \multicolumn{2}{|c|}{ Bandwidth Requirement (kbps) } & \multicolumn{2}{|c|}{ Average ITBG Latency (ms) } & \multicolumn{2}{|c|}{ ITBG Suitability } \\
\hline & & IEEE C37.118.2 & IEC 61850-90-5 & IEEE C37.118.2 & IEC 61850-90-5 & IEEE C37.118.2 & IEC 61850-90-5 \\
\hline Very Low & $<1 \mathrm{pps}$ & $0.77 / 1.66$ & $2.69 / 10.25$ & $11.93 / 13.52$ & $8.28 / 8.65$ & & \\
\hline Low & $1-30 \mathrm{pps}$ & $23.04 / 49.92$ & $80.88 / 307.44$ & $11.93 / 13.52$ & $8.28 / 8.65$ & & \\
\hline Medium & $30-60 \mathrm{pps}$ & $46.08 / 99.84$ & $161.76 / 624.64$ & $11.93 / 13.52$ & $8.28 / 8.65$ & & \\
\hline High & $60-120 \mathrm{pps}$ & $92.16 / 199.68$ & $323.52 / 1259.52$ & $11.93 / 13.52$ & $8.28 / 8.65$ & (1) & \\
\hline
\end{tabular}

Values to the left of ' $/$ are for PMU and to the right are for PDC (containing 5 PMUs data). Blue circle means PMU. Red circle means PDC.

Filled circle means 'Supported'. Half-filled circle means 'Lower required rate bound supported but not the upper bound. Bandwidth requirement considers the upper rate bound.

IEEE C37.118.2 means ITBG-1 input and ITBG-2 output in Fig. 6 are in IEEE C37.118.2 format. IEC 61850-90-5 means both ITBG inputs/outputs are in IEC 61850-90-5 format.

$21.96 \mathrm{~ms}$ average latency does not impact security operations.

Table II presents performance of ITBG protocol translation features. It considers four types of synchrophasor applications (classified based on transmission rate in [17]). Bandwidth requirement depends on type of phasor device, packet size and transmission rate. It can be observed in Table II that bandwidth requirement significantly increases when ITBG output is in IEC 61850-90-5 format. However, this is limitation of IEC 61850-90-5 standard but it is still low enough to allow low latency transmission on today's high capacity access technologies. ITGB protocol translation latencies depend on two factors: (i) complexity of communication standards being inter-converted, (ii) algorithms used to secure synchrophasor packets. Latencies in Table II are averaged over 100 trials. It can be observed that latencies are comparatively higher when IEEE C37.118.2 packets are converted in IEC 6185090-5 packets and vice versa. For conversion from insecure into secure IEC 61850-90-5, latencies are low because ITBG only apply security algorithms but not protocol translation. Based on latency values, ITBG suitability is determine for different types of synchrophasor applications. It is practically validated that ARM based low cost Raspberry Pi is suitable enough to run ITBG software for synchrophasor applications with data transmission rate of up to 100 packets/second. This is reasonably high supported rate then most practical synchrophasor applications.

\section{CONCLUSIONS}

Legacy systems are critical assets in industry. They normally have inherent security vulnerabilities, raise interoperability issues with new systems and are cost prohibitive to replace. Therefore, for smooth transition into secure modernized industry, the proposed low-cost technology bridging solution is of paramount significance for industries.

In contrast to previous works [10], [11], ITBG equips legacy devices and applications with modern and secure technologies. It is a significant refinement over [14] by enabling twoway protocols/standards translation and supporting security features. Moreover, ITBG has modular architectural design that makes it easily customizable for any industrial application.

For validation of ITBG concept, this paper presented implementation and experimental validation of ITBG for synchrophasor applications in smart grid. It successfully enabled interoperability between legacy and new phasor devices/applications by supporting two-way conversion between IEEE
C37.118.2 and IEC 61850-90-5 standards. Further, ITBG ensured secure and trustworthy communication by using GDOI security mechanism and periodically updating security policies and credentials. It is identified that ITBG solution is lightweight, has low resource requirements and suitable to be executed on low-cost ARM based device. Incremental ITBG processing latencies are very small and do not impact realtime transmissions. It is experimentally validated that ARM based ITBG is suitable for most synchrophasor applications with transmission rate of up to 100 packets/second. For very high traffic, ITBG can be deployed on a more powerful device.

\section{REFERENCES}

[1] R. Khan, K. McLaughlin, D. Laverty, and S. Sezer, "Analysis of IEEE C37.118 and IEC 61850-90-5 Synchrophasor Communication Frameworks," in IEEE PES-GM, July 2016.

[2] S. Jaloudi et al., "Communication Strategy for Grid Control and Monitoring of Distributed Generators in Smart Grids using IEC and IEEE Standards," in IEEE ISGT Europe, 2012.

[3] H. P. Breivold and K. Sandstrom, "Internet of Things for Industrial Automation - Challenges and Technical Solutions," in DSDIS, 2015.

[4] A. Ray et al., "Future Research Challenges of Secure Heterogeneous Industrial Communication Networks," in 21st ETFA conference, 2016.

[5] J. Graham, J. Hieb, and J. Naber, "Improving Cybersecurity for Industrial Control Systems," in Int. Symp. on Industrial Electronics, 2016.

[6] R. Khan, K. McLaughlin, D. Laverty, and S. Sezer, "IEEE C37.118-2 Synchrophasor Communication Framework - Overview, Cyber Vulnerabilities Analysis and Performance Evaluation," in ICISSP, 2016.

[7] T. Morris et al., "Cybersecurity Testing of Substation Phasor Measurement Units and Phasor Data Concentrators," in ACM CSIIRW, 2011.

[8] V. K. Singh et al., "Stealthy Cyber Attacks and Impact Analysis on Wide-Area Protection of Smart Grid," in NAPS, 2016.

[9] R. Khan, K. McLaughlin, D. Laverty, and S. Sezer, "Threat Analysis of BlackEnergy Malware for Synchrophasor based Real-time Control and Monitoring in Smart Grid," in 4th ICS-CSR, August 2016.

[10] J. Rosas et al., "Approach to Adapt a Legacy Manufacturing System Into the IoT Paradigm," Interactive Mobile Technologies, vol. 11, 2017.

[11] N. Govindarajan et al., "An Approach for Integrating Legacy Systems in the Manufacturing Industry," in IEEE 14th INDIN, 2016.

[12] P. Priller et al., "Case Study: From Legacy to Connectivity Migrating Industrial Devices into the World of Smart Services," in ETFA, 2014.

[13] T. A. Rizzetti et al., "Cyber Security and Communications Network on SCADA Systems in the Context of Smart Grids," in 50th UPEC, 2015.

[14] S. R. Firouzi et al., "Interpreting and Implementing IEC 61850-905 Routed-Sampled Value and Routed-GOOSE Protocols for IEEE C37.118.2 Compliant Wide-Area Synchrophasor Data Transfer," in Elsevier Journal on Electric Power Systems Research, 2017.

[15] R. Khan, A. Albalushi, K. McLaughlin, D. Laverty, and S. Sezer, "Model based Intrusion Detection System for Synchrophasor Applications in Smart Grid," in IEEE PES-GM, July 2017.

[16] D. M. Laverty et al., "The OpenPMU Project: Challenges and Perspectives," in IEEE PES-GM, 2013.

[17] D. E. Bakken, A. Bose, C. H. Hauser, D. E. Whitehead, and G. C. Zweigle, "Smart Generation and Transmission With Coherent, RealTime Data," Proceedings of the IEEE, vol. 99, no. 6, June 2011. 